\title{
Entanglement as geometry and flow
}

\author{
Sudipto Singha Roy $\odot,{ }^{1}$ Silvia N. Santalla $\odot,{ }^{2}$ Javier Rodríguez-Laguna $\odot,{ }^{3}$ and Germán Sierra ${ }^{1}$ \\ ${ }^{1}$ Instituto de Física Teórica, UAM-CSIC, Universidad Autónoma de Madrid, Cantoblanco, Madrid, Spain \\ ${ }^{2}$ Departemento de Física and Grupo Interdisciplinar de Sistemas Complejos (GISC), Universidad Carlos III de Madrid, Spain \\ ${ }^{3}$ Departemento de Física Fundamental, UNED, Madrid, Spain
}

(Received 19 June 2019; revised manuscript received 1 March 2020; accepted 20 April 2020; published 20 May 2020)

\begin{abstract}
We explore the connection between the area law for entanglement and geometry by representing the entanglement entropies corresponding to all $2^{N}$ bipartitions of an $N$-party pure quantum system by means of a (generalized) adjacency matrix. In the cases where the representation is exact, the elements of that matrix coincide with the mutual information between pairs of sites. In others, it provides a very good approximation, and in all the cases it yields a natural entanglement contour which is similar to previous proposals. Moreover, for one-dimensional conformal invariant systems, the generalized adjacency matrix is given by the two-point correlator of an entanglement current operator. We conjecture how this entanglement current may give rise to a metric entirely built from entanglement.
\end{abstract}

DOI: 10.1103/PhysRevB.101.195134

\section{INTRODUCTION}

Entanglement is one of the most relevant features of the quantum world, constituting the main resource in quantum technologies [1-3] and characterizing the different phases of quantum matter $[4,5]$. In the last years, it has been put forward that even the basic fabric of space-time might be built upon entanglement via the holographic principle and tensor networks [6-12]. Indeed, this suggestive connection stems from the area law: the entanglement entropy of blocks of lowenergy states of local Hamiltonians is frequently proportional to the measure of the boundary separating the block from its environment [13-16], with at most logarithmic corrections [17-19]. Yet, there are relevant exceptions to the area law, such as the rainbow state [20-27] and the Motzkin state [28-34]. In some of these cases, as we will show, an area law is indeed fulfilled for a geometry that differs from the geometry defined by the local structure of the Hamiltonian. Thus, given a quantum state, it is relevant to ask: What is the geometry suggested by the entanglement structure?

Consider a pure state $|\psi\rangle$ of $N$ qubits. There are $2^{N}$ possible subsets or blocks $A \subset \Omega$, and we can compute the von Neumann entropies (alternatively, Rényi entropies) for each, $S_{A}=-\operatorname{Tr}_{A}\left(\rho_{A} \log \rho_{A}\right)$, where $\rho_{A}=\operatorname{Tr}_{\bar{A}}|\psi\rangle\langle\psi|$ and $\operatorname{Tr}_{A}$ and $\operatorname{Tr}_{\bar{A}}$ denote the partial traces on subset $A$ and its complement $\bar{A}$, respectively. Let $I \in\left\{0, \ldots, 2^{N}-1\right\}$ index the subsets through its binary expansion, and let us compute the entanglement entropies for all of them, $\left\{S_{I}\right\}_{I=0}^{2^{N}-1}$. The main question that we will answer is as follows: Does this set of entropies respond to an area law for some geometry?

This article is organized as follows. In Sec. II we define the entanglement adjacency matrix (EAM), which explains the geometry encoded in the entanglement data, while its properties are discussed in Sec. III. Some exact examples are discussed in Sec. IV. The cases, for which we must recourse to numerical computations, are detailed in Sec. V. The EAM provides a route to define a generic entanglement contour, as is shown in Sec. VI. Interestingly, from the point of view of conformal field theory (CFT), the EAM entries can be written as the two-point correlator of an entanglement current, and many of their properties can be readily understood. Based on the CFT insight, we ask whether an entanglement metric can be defined from the EAM, and a conjecture in that direction is provided in Sec. VIII. The article concludes in Sec. IX with a list of our conclusions and our proposals for further work.

\section{DEFINING A GEOMETRY: ENTANGLEMENT ADJACENCY MATRIX}

With the purpose of investigating the area law, we shall define a geometry through an adjacency matrix $J$ such that $J_{i j}>0$ when sites $i$ and $j$ are somehow connected or zero otherwise $\left(J_{i j}=J_{j i}\right)$. The von Neumann entropy of a subset $A \subset \Omega$ will be given by the sum of the weights corresponding to the broken links

$$
S_{A}=\sum_{i \in A} \sum_{j \in \bar{A}} J_{i j},
$$

of course, Rényi entropies of order $n$ can be employed, thus defining $S_{A}^{(n)}$ and $J_{i j}^{(n)}$. Alternatively, a constant $s_{0}$ term may be added to the right-hand side (rhs) of Eq. (1), which may constitute a topological entropy term [35]. If Eq. (1) holds, matrix $J$ will be termed the entanglement adjacency matrix (EAM) of the state. Notice that $J_{i j}$ is the entanglement entropy that we gain by separating node $i$ from node $j$. A schematic representation of the above formulation is presented in Fig. 1. Additionally, the $J_{i j}$ 's may be employed to build a notion of distance, or an entanglement metric, along recent proposals $[11,12]$, that we will discuss in Sec. VIII.

Figure 2 presents a different way to conceptualize the entropy of any bipartition, assuming the validity of Eq. (1), similar to the Venn diagrams used in classical information 


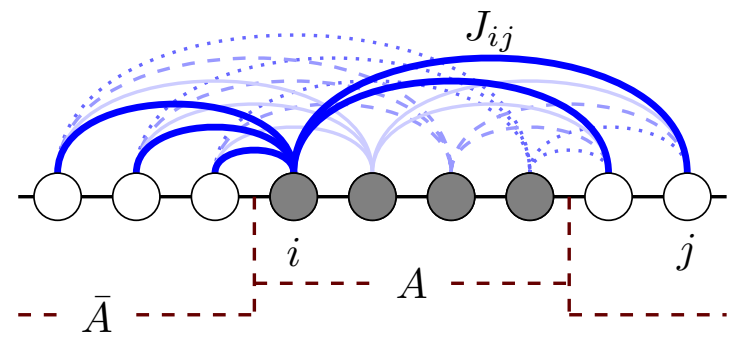

FIG. 1. Schematic of the entanglement entropy obtained for an arbitrary bipartition $(A, \bar{A})$ by adding up the links connecting the sites. Here the link intensities represent the EAM entries $J_{i j}$.

theory [36]. Let the square represent the whole $J$ matrix. Then the entropy of blocks $A$ or $B$ is found by counting the shaded elements of Figs. 2(a) or 2(b), which are the ones that connect the blocks with their compliments. But these quantum versions of Venn diagrams can convey more information. The mutual information between blocks $A$ and $B$, defined as $\mathcal{J}(A$ : $B)=S(A)+S(B)-S(A \cup B)$, which is twice the shaded area in Fig. 2(c), i.e., the matrix elements connecting blocks $A$ and $B$.

\section{PROPERTIES OF THE ENTANGLEMENT ADJACENCY MATRIX}

Before considering the validity of Eq. (1) for the quantum states of physical interest, let us list a few relevant properties of the elements of the EAM, $J_{a b}$.

\section{A. Positivity: $J_{a b} \geqslant 0$}

Proof. The entanglement entropy of site $a$ can be easily obtained (assuming $s_{0}=0$ ): $S_{a}=\sum_{j \neq a} J_{a j}$. Similarly, the entropy of a block composed by sites $a$ and $b$ is given by

$$
S_{a b}=\sum_{j \neq a, b}\left(J_{a j}+J_{b j}\right)=S_{a}+S_{b}-2 J_{a b} .
$$

Thus, we can find the mutual information between sites $a$ and $b, \mathcal{J}(a: b)$,

$$
\mathcal{J}(a: b) \equiv S_{a}+S_{b}-S_{a b}=2 J_{a b}
$$

(a)

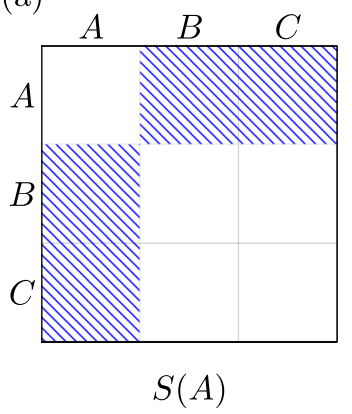

(b)

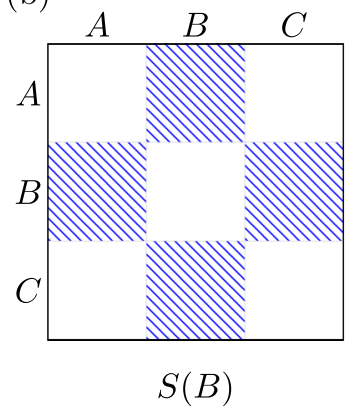

thus providing a simple physical interpretation for $J_{a b}$ as the mutual information of the pair of sites $a, b$. Mutual information must be positive, thus reinforcing our notion that $J_{a b} \geqslant 0$ must hold (if $s_{0}=0$ ).

This interpretation of the entries of the entanglement adjacency matrix as mutual information of sites yields the next corollary.

Corollary: If Eq. (1) is exact, knowledge about the entanglement of all single sites and all pairs of sites is enough to determine the entanglement of all blocks.

\section{B. Subadditivity condition}

The entanglement entropies obtained using the elements of entanglement adjacency matrices fulfill certain subadditivity conditions as follows.

Given any three subsets, $A, B$, and $C \subset \Omega$, the strong subadditivity condition must hold [36]

$$
S_{A B}+S_{B C} \geqslant S_{A B C}+S_{B} .
$$

Whenever Eq. (1) holds with $J_{a b} \geqslant 0$, even with $s_{0} \neq 0$, this inequality will hold also.

Proof. First of all, let us consider the blocks $A, B$, and $C$ contain a single site, respectively, $a, b$, and $c$. Then we can prove the following:

$$
S_{a b}+S_{b c} \geqslant S_{a b c}+S_{b},
$$

which is the site equivalent of Eq. (4). To prove the above relation, we can compute each of the terms

$$
\begin{aligned}
S_{b} & =\sum_{j \neq b} J_{b j}, \\
S_{a b} & =\sum_{j \neq a, b}\left(J_{a j}+J_{b j}\right), \\
S_{b c} & =\sum_{j \neq b, c}\left(J_{c j}+J_{b j}\right), \\
S_{a b c} & =\sum_{j \neq a, b, c}\left(J_{a j}+J_{b j}+J_{c j}\right),
\end{aligned}
$$

FIG. 2. Illustrating the structure of the entanglement adjacency matrix through Venn diagrams. (a) Evaluation of the entanglement entropy of block $A$ requires adding up all $J_{i j}$ elements joining $A$ to its complement $(\bar{A}=B \cup C)$, given by the shaded area. (b) The same situation holds for block $B$. (c) The mutual information of blocks $A$ and $B, \mathcal{J}(A: B)=S(A)+S(B)-S(A \cup B)$ is (twice) the darker area shaded in this panel, given by the sum of the matrix entries $J_{i j}$ joining both blocks. 

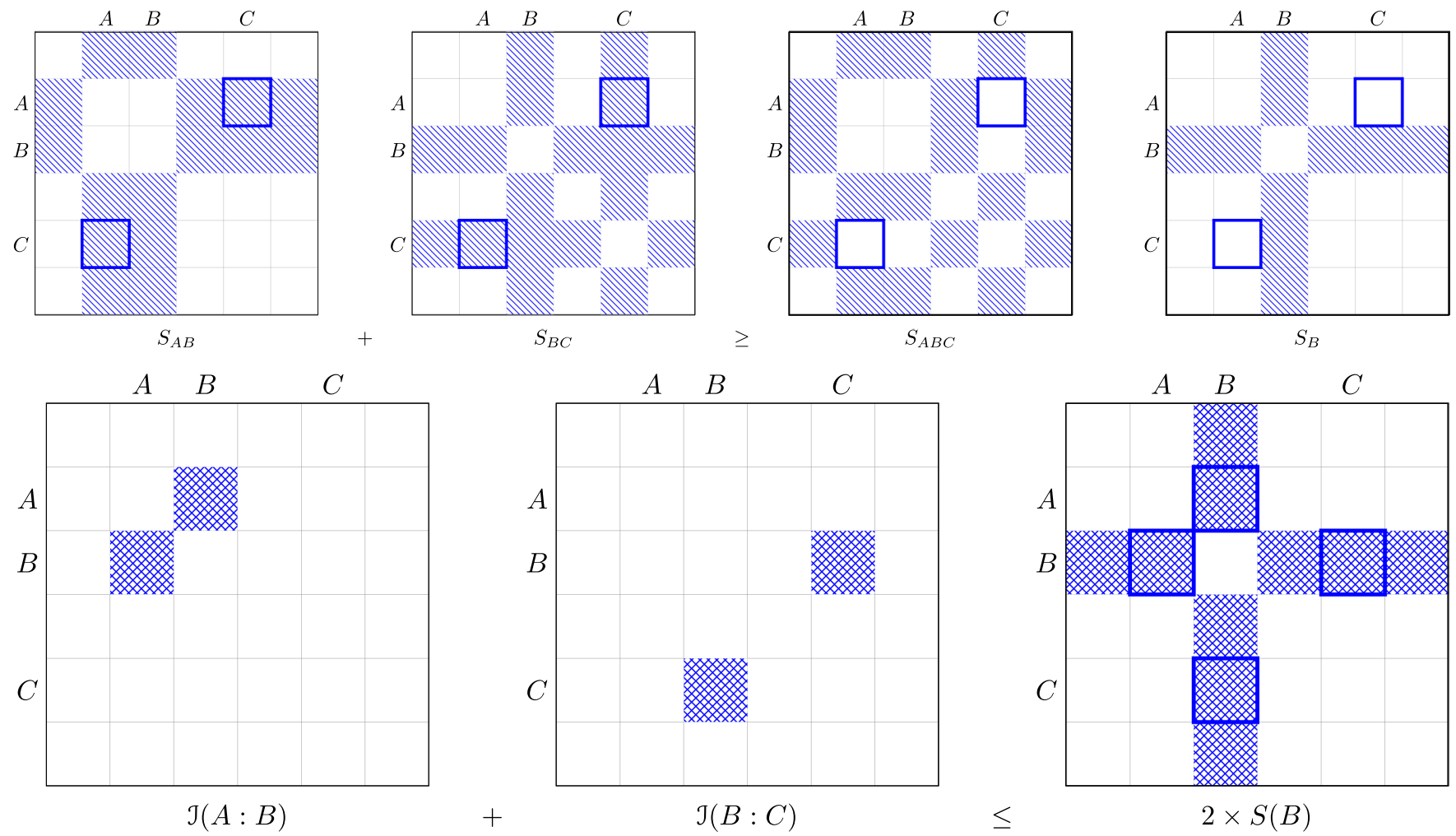

FIG. 3. Top: Graphical illustration of the validity of Eq. (4). The marked squares, filled on the lhs and empty on the rhs, account for the inequality. Bottom: Graphical illustration of the validity of Eq. (9). The marked squares on the rhs are the filled squares on the lhs of the inequality.

from which we derive

$$
\begin{aligned}
S_{a b}+S_{b c}= & \sum_{j \neq a, b} J_{a j}+\sum_{j \neq a, b} J_{b j}+\sum_{j \neq b, c} J_{b j}+\sum_{j \neq b, c} J_{c j} \\
= & \sum_{j \neq a, b, c}\left(J_{a j}+J_{b j}+J_{b j}+J_{c j}\right) \\
& +J_{a c}+J_{b c}+J_{a b}+J_{a c} \\
= & S_{a b c}+\sum_{j \neq a, b, c} J_{b j}+2 J_{a c}+J_{b c}+J_{b a} \\
= & S_{a b c}+S_{b}+2 J_{a c} .
\end{aligned}
$$

Due to the positivity of $J_{a c}$, the strong subadditivity condition is therefore proved.

Now let us consider general subsets $A, B$, and $C \subset \Omega$. We can additionally prove the following important lemma.

Lemma: A partition of the nodes into blocks leads to an effective entanglement adjacency matrix neglecting the intrablock links and adding the interblock ones. In other terms, if we define blocks $\left\{B_{k}\right\}$, with $B_{k} \subset \Omega, B_{k} \cap B_{l}=\emptyset$ if $k \neq l$, $\cup_{k} B_{k}=\Omega$, then we can define

$$
J_{k l}^{B} \equiv \sum_{\substack{a \in B_{k}, b \in B_{l}}} J_{a b},
$$

if $k \neq l$ and $J_{k k}^{B}=0$. Then Eq. (1) still holds for partitions which do not break the blocks. The effective entanglement adjacency matrix entries retain their physical meaning of mutual information. Similarly, the site entropies $S_{a}, S_{a b}, S_{b c}, S_{a b c}$ can be generalized to corresponding block entropies $S_{A}, S_{A B}$, $S_{B C}, S_{A B C}$. Using this lemma, and the relation in Eq. (7) we can prove the strong subadditivity condition of three subsets of $\Omega, A, B$, and $C$, given in Eq. (4).

A graphical illustration of Eq. (4) can be seen in Fig. 3, along with an illustration of a direct variant

$$
\mathcal{J}(A: B)+\mathcal{J}(B: C) \leqslant 2 S(B) .
$$

\section{Recursion relation for entropies of contiguous blocks}

If the quantum state is translationally invariant in onedimension (1D), a recursion relation that generalizes Eq. (3) can be proved for entropies of contiguous blocks $S_{l}$. Since $J_{i j}=J(l=|i-j|)$, we have

$$
J_{l}=S_{l}-\frac{1}{2} S_{l-1}-\frac{1}{2} S_{l+1} .
$$

The proof can be obtained by the mere substitution of Eq. (1).

We may now ask the following question: Are there any real quantum states for which expression (1) is exact or, at least, approximate? In the forthcoming sections, we carry out analytical and numerical analysis to answer this question in detail.

\section{EXACT EXAMPLES}

To obtain the entanglement adjacency matrix for any general pure quantum many-body state, one needs to compute the entanglement of all possible bipartitions of the state, which increases exponentially with the size of the system. Hence, computation of the entanglement adjacency matrix, which 
(a)

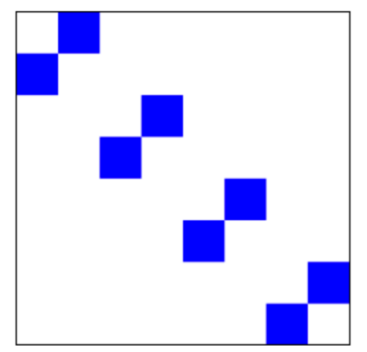

(b)

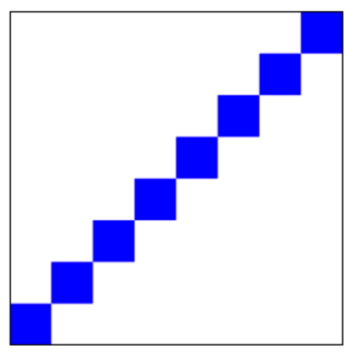

FIG. 4. Graphical representation of the entanglement adjacency matrices obtained analytically for (a) the dimer state and (b) the rainbow state. Here $N=8$.

provides even approximate representation of all the entropies, often requires some computational effort. We discuss the methodology in detail in Sec. V. However, in some cases in which the entropies of the quantum state can be computed analytically, the corresponding entanglement adjacency matrix can be obtained straightforwardly. Below we discuss a few such cases.

\section{A. Valence bond states}

Let us consider valence bond states, where qubits are paired into maximally entangled Bell pairs $|\Psi\rangle=\left|i_{1}, j_{1}\right\rangle \otimes$ $\cdots \otimes\left|i_{m}, j_{m}\right\rangle$, with $m=N / 2$ (for even $N$ ) and $|i, j\rangle=\frac{1}{\sqrt{2}}(\mid+$ $-\rangle_{i j} \pm|-+\rangle_{i j}$ ). In that case, Eq. (1) represents exactly the entanglement entropy of every partition, as long as $J_{i j}=\log 2$ iff $i=i_{k}$ and $j=j_{k}$ (or vice versa) for some $k$, and zero otherwise. Interestingly, such states approximate the ground states (GS) of a strongly inhomogeneous free-fermionic Hamiltonian

$$
H_{\mathrm{f}-\mathrm{f}}=-\frac{1}{2} \sum_{i, j=1}^{N} t_{i j} c_{i}^{\dagger} c_{j},
$$

where $c_{i}$ stands for the annihilation operator for a spinless fermion on site $i$ and $t_{i j}$ are hopping amplitudes. Below we consider two important members of the valence bond states, which can be derived from the above Hamiltonian at certain limits of the hopping term $t_{i j}$.

\section{Dimer-model}

If we set $t_{i, i+1}=\left[1+(-1)^{i+1} \delta\right]$, and all other $t_{i j}=0$, for $\delta \lesssim 1$, the GS will approximate the dimer state whose $J_{i j}=$ $\log 2$ only for $i=2 k-1$ and $j=2 k$, for $k=1,2, \ldots, \frac{N}{2}$, which is still tridiagonal, resulting in a mere restriction of the one-dimensional adjacency matrix representing the Hamiltonian. A graphical representation of the same is depicted in Fig. 4(a).

\section{Rainbow chain}

Another important member of the family of valence bond states, the rainbow state, can be derived from the Hamiltonian in Eq. (11), for the following choices of the system parameters, $t_{\frac{N}{2}, \frac{N}{2}+1}=1$ and $t_{i, i+1}=\exp [-h(|i-N / 2|-1 / 2)]$, for $h \gg 1$. In this case, the Bell pairs are established among symmetric qubits with respect to the center: $i_{k}=k, j_{k}=$ $N+1-k[20,21]$. Thus, all the entanglement entropies of

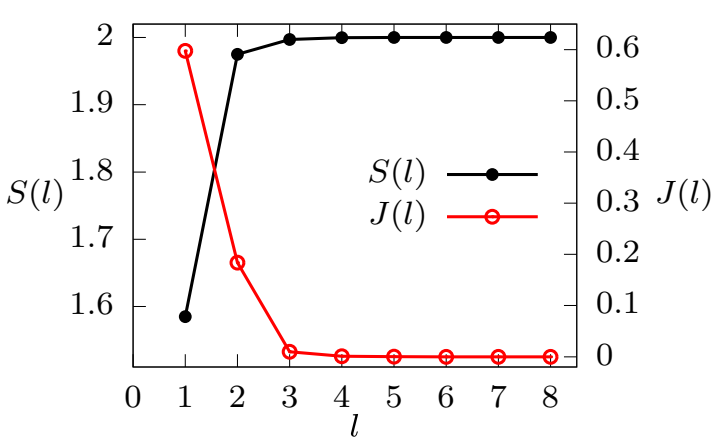

FIG. 5. Plot of $S(l)$ given in Eq. (12), along with the $J(l)$ obtained using Eq. (12) into Eq. (10). Both results are expressed taking logs with a base of 2 .

the rainbow state are reproduced by Eq. (1) using $J_{i j}=\log 2$ iff $i+j=N+1$, and zero otherwise. Similar to the previous example, a graphical representation of the $J$-matrix obtained in this case is given in Fig. 4(b). Notice that, in the rainbow case, the entanglement adjacency matrix is not emerging as a restriction on the adjacency matrix representing the Hamiltonian. In other words, an observer trying to determine the geometry from observations of the entanglement will not find the geometry of the Hamiltonian.

\section{B. Affleck-Kennedy-Lieb-Tasaki (AKLT) state}

Let us now consider a translational invariant quantum state, the Affleck-Kennedy-Lieb-Tasaki (AKLT) state on a spin-1 chain [37], defined by its matrix product form built on the Pauli matrices, $A_{k}=\sigma_{k}$, and derive the exact expression of the $J(l=|i-j|)$ as follows. The expression of entanglement entropy for a block of $l$ consecutive sites obtained from the periodic AKLT state with $N \gg 1$ is given by [38-40]

$$
\begin{aligned}
S_{l}= & \log 4-\frac{3}{4}\left(1-p^{l}\right) \log \left(1-p^{l}\right) \\
& -\frac{1}{4}\left(1+3 p^{l}\right) \log \left(1+3 p^{l}\right),
\end{aligned}
$$

where $p=-\frac{1}{3}$. For $l \gg 1, S_{l}$ approaches asymptotically the value $2 \log 2$ that corresponds to cutting the valence bonds that connect the block to the rest of the system (see Fig. 5). $J(l)$ can be found by plugging Eq. (12) into the recursion relation for translationally invariant states, Eq. (10). The result is plotted in Fig. 5. Notice the fast decreases of $J(l)$ with $l$, that can be obtained expanding the entropies in Eq. (12) as

$$
\begin{aligned}
S_{l} \approx & \log 4-\frac{3}{4}\left(1-p^{l}\right)\left[-p^{l}-\frac{1}{2}\left(p^{l}\right)^{2}\right] \\
& -\frac{1}{4}\left(1+3 p^{l}\right)\left[3 p^{l}-\frac{1}{2}\left(3 p^{l}\right)^{2}\right] . \\
\approx & \log 4-\frac{3}{2} p^{2 l} .
\end{aligned}
$$

Replacing this into Eq. (10), and only keeping the terms up to $p^{2 l}$, we get

$$
\begin{aligned}
J_{l}= & \log 4-\frac{3}{2} p^{2 l}-\frac{1}{2} \log 4+\frac{1}{2}\left(\frac{3}{2} p^{2} p^{2 l}\right) \\
& -\frac{1}{2} \log 4+\frac{1}{2}\left(\frac{3}{2} p^{-2} p^{2 l}\right),
\end{aligned}
$$

which after simplification yields

$$
J_{l} \propto p^{2 l} .
$$


Therefore, for the AKLT state, $J_{l}$ decays faster than that of spin-correlation function $\left\langle\vec{S}_{0} \vec{S}_{l}\right\rangle=4 p^{l}$ [37].

\section{GHZ state}

A different case is provided by the Greenberger-HorneZeilinger (GHZ) state, $|\psi\rangle_{\mathrm{GHZ}}=\frac{1}{\sqrt{2}}\left(|0\rangle^{\otimes N}+|1\rangle^{\otimes N}\right)$. In this case, the entanglement entropy of all partitions is equal to $\log 2$. It can be proved that Eq. (1) can only represent this situation making $J_{i j}=0$ for all $i, j$ and $s_{0}=\log 2$. This amounts to the fact that the GHZ state does not have a geometrical interpretation in this framework.

\section{NUMERICAL COMPUTATION OF THE ENTANGLEMENT ADJACENCY MATRIX}

Equation (1) attempts to represent $2^{N}$ entanglement entropy values using only $N_{p}=N(N-1) / 2$ parameters (neglecting $s_{0}$ ). The relation between parameters and entropies is linear, expressed through

$$
\sum_{(i j)} \mathcal{A}_{I,(i j)} J_{i j}=S_{I}
$$

where $I=\left(x_{1}, \ldots, x_{N}\right)$ denotes the binary expansion for the index of each block, i.e., $x_{k}=1$ if site $k$ belongs to block $I$ (and zero otherwise), and $\mathcal{A}$ is a $2^{N} \times N_{p}$ matrix with entries given by $\mathcal{A}_{\left(x_{1}, \ldots, x_{N}\right),(i j)}=1$ if $\left(x_{i}, x_{j}\right)=(0,1)$ or $(1,0)$, and zero otherwise. Equation (16) is a strongly overdetermined linear system which will be, in general, incompatible. Yet it is possible to find an approximate solution in the least-squares sense, using the so-called normal equations

$$
\sum_{\left(i^{\prime} j^{\prime}\right)}\left(\mathcal{A}^{\dagger} \mathcal{A}\right)_{(i j),\left(i^{\prime} j^{\prime}\right)} J_{i^{\prime} j^{\prime}}=\sum_{I} \mathcal{A}_{I,(i j)} S_{I}
$$

So element $k l$ of matrix $\mathcal{A}^{T} \mathcal{A}$ provides the number of blocks which break both index $k$ and index $l$. This number is independent of $k$ and $l$ as long as $k \neq l$. For a system with $N$ sites, the number of blocks which break a given coupling is always the same, $2^{N-1}$. The number of blocks which break two given couplings is also the same: $2^{N-2}$. Thus matrix $\mathcal{A}^{T} \mathcal{A}$ is given by

$$
\mathcal{A}^{T} \mathcal{A}=2^{N-2}\left[\begin{array}{ccccc}
2 & 1 & 1 & \ldots & 1 \\
1 & 2 & 1 & \ldots & 1 \\
1 & 1 & 2 & \ldots & 1 \\
\vdots & \vdots & \vdots & \ddots & \\
1 & 1 & \ldots & \ldots & 2
\end{array}\right]
$$

Equation (17) is a linear system of $N_{p}$ equations for $N_{p}$ unknowns with a unique solution, but the computational cost is still exponential because it requires the evaluation of $2^{N}$ entropies. Nevertheless, an approximate solution can be found using a random sample of the total set of entropies.

We quantify the relative error made in the optimization process described above, as follows. Let $\hat{S}_{I}$ be the estimate obtained through Eq. (17). The error will be defined as

$$
\mathcal{E}=\frac{1}{2^{N}} \sum_{I=0}^{2^{N}-1}\left|S_{I}-\hat{S}_{I}\right|
$$

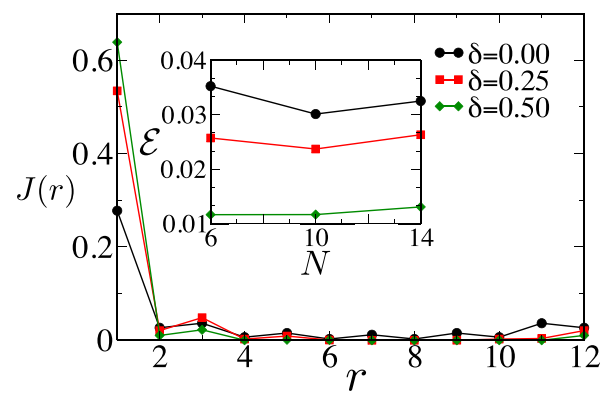

FIG. 6. We consider the nearest-neighbor dimerized Hamiltonian with periodic boundary condition and $N=14$. Plots of $J(r)=J_{2,2+r}$ with the distance $r$ are obtained for different $\delta$ values. Additionally, in the inset, we plot the scaling of the error function $\mathcal{E}$ with the system size $N$, for the same choices of the parameters.

We will use this formula to estimate the error made in the computation of the entanglement adjacency matrix for certain physical models we consider below.

\section{A. Free-fermionic model}

Let us consider free-fermionic systems, as described in Eq. (11). The GS of Eq. (11) is a Slater determinant built from the lowest-energy eigenstates of the hopping matrix $t_{i j}$. Let $U_{k, i}$ be the matrix containing such eigenstates as columns. Then

$$
C_{i j}=\left\langle c_{i}^{\dagger} c_{j}\right\rangle=\sum_{k \in K} \bar{U}_{k, i} U_{k, j},
$$

where $K$ denotes the set of occupied orbitals, which we will assume to be the half with negative energies. The von Neumann entropy for a block $A$ is found from the eigenvalues of the restriction of $C_{i j}$ to that block, $v_{p} \in[0,1][41]$

$$
S_{A}=\sum_{p=1}^{|A|} H\left(v_{p}\right), \quad H(x)=-[x \log x+(1-x) \log (1-x)] .
$$

Once the entropy values are computed, the optimal entanglement adjacency matrix can be obtained by solving the set of linear Eqs. (17). In Fig. 6 we plot the $J(r)=J_{2,2+r}$ with the distance $r$, for the dimerized Hamiltonian, which can be derived from the free-fermionic model expressed in Eq. (11), for $t_{i, i+1}=1+(-1)^{i} \delta$. From the figure, we can see that starting from a high value, $J(r)$ decreases with $r$. Moreover, $J(r)$ presents slowly decaying parity oscillations. A true short-ranged behavior emerges as the GS configuration tends towards the dimer configuration for higher values of $\delta$. Subsequently, we present a scaling of the error $(\mathcal{E})$ estimated in all these cases with the system size $N$. The maximum error turns out to be $<4 \times 10^{-2}$. Though $\mathcal{E}$ quantifies the average error introduced in the computation of entropy of any bipartition, a more fine-grained analysis of the error, in this case, reveals the fact that the amount of error is relatively lower in the case of bipartitions with a lesser number of boundaries (see Appendix A for a detailed discussion). 


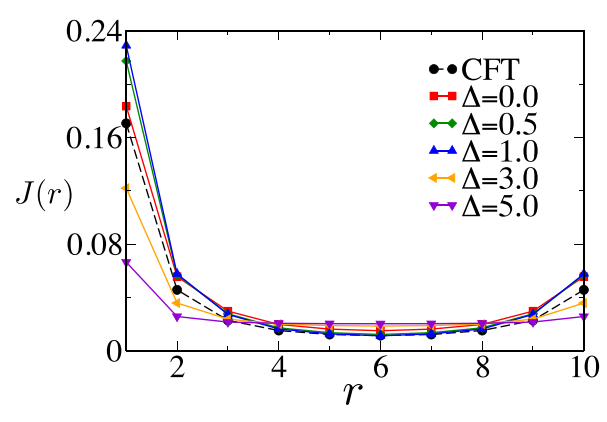

FIG. 7. We obtain the same profiles for the $X X Z$ model expressed in Eq. (22), with $N=12$ and for different values of the anisotropy parameter $\Delta$. The broken black line corresponds to the analytical expression $J(r)=\frac{1}{6}\left(\frac{\pi}{N}\right)^{2} \frac{1}{\sin ^{2}(\pi r / N)}$, obtained for the conformal case (see Table I in Appendix C).

\section{B. Interacting model: $X X Z$ Hamiltonian}

We next discuss the optimal entanglement adjacency matrix obtained for an interacting model, the $X X Z$ model in $1 \mathrm{D}$ with periodic boundaries, expressed as

$$
H_{X X Z}=\sum_{i}^{N}\left[S_{i}^{x} S_{i+1}^{x}+S_{i}^{y} S_{i+1}^{y}+\Delta S_{i}^{z} S_{i+1}^{z}\right],
$$

where $S_{i}^{k}(k \in x, y, z)$ are the spin- $1 / 2$ operators at site $i$, and $\Delta$ denotes the anisotropy constant. We carry out the same analysis as before and obtain the entanglement adjacency matrix by solving the set of linear Eqs. (17). However, in this case, the entropy values cannot be obtained from the correlation matrix. Rather, we need to perform an exact diagonalization and obtain the reduced density matrices directly from the GS itself. The behavior of $J(r)=J_{2,2+r}$ with the distance $r$ is depicted in Fig. 7, for the following values of the anisotropy parameter $\Delta=0.0,0.5,1.0$ (critical cases), and 3.0,5.0 (noncritical cases in the gapped antiferromagnetic phase).

In general, spin and the fermion representations lead to different reduced density matrices for nonconsecutive blocks [42]. In our case, to obtain the optimized geometry, we must consider both consecutive and nonconsecutive blocks, a clear difference emerges in the behavior of $J(r)$ obtained in this case. We observe that unlike the fermionic case, the profile of $J(r)$ obtained using the spin representation does not exhibit the usual parity oscillations. In this case, the maximum error $(\epsilon)$ computed using the formula in Eq. (19), turns out to be $\sim 6 \times 10^{-2}$. Moreover, here the random sampling of only $5 \times$ $10^{2}$ entropies provides close agreement with the above results (see Fig. 10 in Appendix A). Hence, the random sampling method can ensure the scalability of the formalism and unveil important features at larger system sizes, when exploring all possible bipartitions becomes impracticable.

\section{ENTANGLEMENT CONTOUR}

In the subsequent part of our analysis we use the entanglement adjacency matrix to refine the entanglement structure contained in the block entropies, making use of the entanglement contour function [43]. The entanglement contour for a given block $A$, introduced by Chen and Vidal [43] and analyzed in several other works [24,44-49], is a positive partition of the entanglement entropy associated to the block sites, i.e., a function $s_{A}(i)$ with $i \in A$, such that

$$
S_{A}=\sum_{i \in A} s_{A}(i), \quad s_{A}(i) \geqslant 0 .
$$

Interestingly, the entanglement adjacency matrix provides a natural entanglement contour, using expression (1),

$$
s_{A}(i) \equiv \sum_{j \in \bar{A}} J_{i j} .
$$

Furthermore, using the properties of $J_{i j}$ described in Sec. III, it can be shown that our proposal for the entanglement contour function satisfies all the constraints listed in Ref. [43]. Below we list a few of those relevant properties.

(1) Positivity: $s_{A}(i) \geqslant 0$.

Equation (3) guarantees the positivity of the elements of the entanglement adjacency matrix $J_{i j}$, which implies that the contour function $s_{A}(i)$ defined in Eq. (24) must be positive.

(2) Normalization: $\sum_{i \in A} s_{A}(i)=S_{A}$.

From Eqs. (1) and (24), we obtain the entropy of the subset $A$ is given by $S_{A}=\sum_{i \in A} \sum_{j \in \bar{A}} J_{i j}=\sum_{i \in A} s_{A}(i)$. Hence, the normalization condition of the contour function is guaranteed.

(3) Symmetry: If $\mathcal{F}$ is a symmetry of $\rho_{A},\left(\mathcal{F} \rho_{A} \mathcal{F}^{\dagger}=\rho_{A}\right)$ that exchanges the sites $i$ and $j$, then $s_{A}(i)=s_{A}(j)$.

This statement applies to space reflections, translations, and rotations. In our case, the symmetry must apply to the whole wave function $|\psi\rangle_{A B}$, not to the reduced density matrix of a single block, since the $J_{i j}$ 's is a property of the entire set of partitions. A symmetry of the wave function will be reflected in a symmetry of the set of entanglement entropies of the different blocks. The solution to the normal equations, Eq. (17), is unique and must reflect the symmetries of the wave function.

Proof of properties viz. the invariance of the entanglement contour under local unitaries and its upper and lower bounds are given in Appendix B.

For free-fermion models, a proposal for the contour is [43]

$$
s_{A}(i)=\sum_{p=1}^{|A|}\left|\Phi_{p, i}^{(A)}\right|^{2} H\left(v_{p}\right),
$$

where $\Phi_{p, i}^{(A)}$ is the eigenvector, with eigenvalue $v_{p}$, of the correlation matrix (20) restricted to the block $A$. In this regard, here, we stress the fact that unlike the above formulation, our approach aims to provide an entanglement contour function by considering contributions of all bipartitions and not just the ones consisting of simply connected intervals. Moreover, the formalism can be applied to any general quantum system, including the interacting models. Figure 8 shows that the contours (24) and (25) for a free-fermion model are very similar [50].

\section{ENTANGLEMENT CURRENT}

The entanglement entropy of the GS of a conformal field theory (CFT) for an interval $A=(u, v)$ embedded in the infinite line is given by [17-19]

$$
S_{A}=\frac{c}{3} \log \frac{v-u}{\varepsilon},
$$




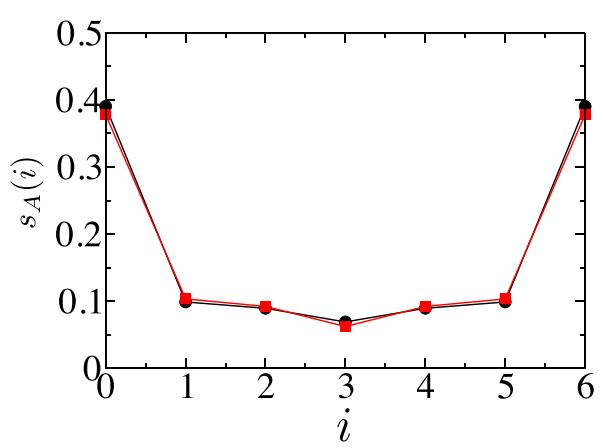

FIG. 8. Comparison of the contour functions for the entanglement entropy $s_{A}(i)$, obtained for the half-chain of a clean freefermionic system $\left[t_{i j}=1,|i-j|=1\right.$ in Eq. (11)], using Eqs. (24) (red squares) and (25) (black circles). Here we consider $N=14$.

where $c$ is the central charge and $\varepsilon>0$ a short distance cutoff. Equation (26) can be obtained from a continuous version of Eq. (1)

$$
S_{A}=\int_{A_{\varepsilon}} d x \int_{B} d y J(x, y),
$$

with $A_{\varepsilon}=(u+\varepsilon, v-\varepsilon)$ and $B=(-\infty, u) \cup(v, \infty)$, by choosing

$$
J(x, y)=\frac{c / 6}{(x-y)^{2}} .
$$

Note that the above choice of the entanglement adjacency matrix is also emanating from the recursion relation given in Eq. (10), when the continuum limit is considered. In particular, if we expand the entropies obtained for the contiguous blocks $S_{l}$, we get $J_{l}=-\frac{1}{2} \frac{d^{2} S_{l}}{d l^{2}}$. Equation (28) indicates that $J(x, y)$ is the two-point correlator, on the plane of the spatial component of a current operator $\mathbf{J}$, whose integration along segments, as in Eq. (27), is invariant under reparametrizations. $J(x, y) d x d y$ represents the amount of entanglement between the intervals $(x, x+d x)$ and $(y, y+d y)$. This interpretation of $\mathbf{J}$ holds in more general situations. Indeed, using the construction by Cardy and Tonni for entanglement Hamiltonians in CFT [51], we can show that Eq. (27) reproduces the values of $S_{A}$, for the space-time geometries $\Sigma$, that are conformally equivalent to an annulus. In these cases $J(x, y)$ is given by the two-point correlator (see Appendix C)

$$
J(x, y)=\langle\mathbf{J}(x) \mathbf{J}(y)\rangle_{\Sigma} .
$$

The origin of the entanglement current $\mathbf{J}$ can be traced back to the way the $n$th Rényi entropy, $S_{A}^{(n)}=\frac{1}{1-n} \log \operatorname{tr}_{A} \rho_{A}^{n}$, is computed using twist fields $[19,52,53]$. For the interval $A=$ $(u, v)$ in the infinite line one has

$$
\operatorname{tr}_{A} \rho_{A}^{n}=\left\langle\overline{\mathcal{T}}_{n}(u) \mathcal{T}_{n}(v)\right\rangle=\frac{c_{n}}{[(v-u) / \varepsilon)]^{2 \Delta_{n}}},
$$

where $\mathcal{T}_{n}(x)$, and its conjugate $\overline{\mathcal{T}}_{n}(x)$, are twist fields with the same scaling dimension $\Delta_{n}=\frac{c}{12}(n-1 / n)$, and $c_{n}$ is a nonuniversal constant whose value at $n=1$ is $c_{1}=1$ to guarantee the normalization condition $\operatorname{tr}_{A} \rho_{A}=1$. The von Neumann entropy $S_{A}$, given in Eq. (26), can be derived as $\lim _{n \rightarrow 1} S_{A}^{(n)}$ using Eq. (30). There is also an additive constant constant $c_{1}^{\prime}$, not included in Eq. (26), that comes from the derivative of $c_{n}$ at $n=1$.

The relation between the entanglement current and the twist fields is based on Eq. (30). Taking derivatives respect to the coordinates $u$ and $v$ one finds, in the limit $n \rightarrow 1$

$$
\lim _{n \rightarrow 1} \frac{1}{2(1-n)}\left\langle\partial_{u} \overline{\mathcal{T}}_{n}(u) \partial_{v} \mathcal{T}_{n}(v)\right\rangle=\frac{c / 6}{(v-u)^{2}},
$$

that compared to Eq. (28) yields the formal identification

$$
\mathbf{J}(x)=\lim _{n \rightarrow 1} \frac{1}{\sqrt{2(1-n)}} \partial_{x} \mathcal{T}_{n}(x),
$$

and the same expression with $\overline{\mathcal{T}}_{n}(x)$. It is important to observe that exchanging the limit $n \rightarrow 1$ and the $u$ and $v$ derivatives in Eq. (31) is not well defined since $\lim _{n \rightarrow 1}\left\langle\overline{\mathcal{T}}_{n}(u) \mathcal{T}_{n}(v)\right\rangle /(1-$ $n)=\infty$. There is no a priori reason why these two operations should commute. The order of operations we chose in Eq. (31) is perfectly consistent and allow us to identify the entanglement current (32). This derivation is certainly different from the standard one where the entanglement entropy is found by taking the logarithm of Eq. (30) and the limit $n \rightarrow 1$. Notice that the twist fields become the identity in the limit $n \rightarrow 1$, so their derivative are fields of dimension 1 .

Applying Eqs. (27) and (28) to disconnected intervals on a line gives the formula derived for $S_{A}$ in Ref. [19] (see Appendix C), but misses a term that depends on the harmonic ratio of the entangling points [54-60]. The current operator can be written as $J_{\mu}=\partial_{\mu} \phi$, where $\phi$ is a massless boson, that implies its conservation, i.e., $\partial_{\mu} J_{\mu}=0$. If the scalar field $\phi$ has a mass $m$, one can derive from Eq. (27) the entropy $S_{A} \simeq \frac{c}{3} \log (\xi / \varepsilon)$ that corresponds to a massive field theory in the scaling limit with correlation length $\xi=1 / m$ [19]. Equations (27) and (29) can be generalized to models in $D$ spatial dimensions recovering the area law for $S_{A}$.

Equations (3) and (28) seem to suggest that the mutual information $\mathcal{J}(x: y)$ in CFT has a universal scaling behavior $|x-y|^{-2}$. This property holds for the free-fermion model studied above, but not in general. A recent example was studied in Ref. [61], when $\mathcal{J}(x: y)$ is computed for certain spin-Hamiltonians. In this case, it was reported that $\mathcal{J}(x: y) \propto$ $|x-y|^{-\eta}$ with $\eta=1 / 2$ for the critical Ising model, and $\eta=1$ for the $X X$ spin model [61]. The later results do not contradict Eq. (28) since the spin representation may exhibit a different decay rate of bipartite entanglement [42].

\section{TOWARDS AN ENTANGLEMENT METRIC}

Let us consider the intriguing possibility that the $J_{i j}$ might be employed to build a metric entirely based on entanglement properties, as other authors have recently explored [11,12]. The basic assumption is that highly entangled sites are, somehow, nearby, while disentangled sites are further away. We suggest that $J_{i j}$ determines univocally a single-step distance between sites $i$ and $j$ through an unknown function

$$
d_{i j}=\Phi\left(J_{i j}\right)
$$

which is monotonously decreasing and fulfills $\Phi(0) \rightarrow \infty$ and $\Phi\left(J_{\max }\right)=\ell_{0}$, where $J_{\max }$ is the maximal possible value of $J$ (for qubits, $J_{\max }=\log 2$ ) and $\ell_{0}$ is the minimal length 
scale. A reasonable choice, following our CFT discussion, is

$$
\Phi(J)=\ell_{0}\left(J / J_{\max }\right)^{-1 / 2} .
$$

In analogy to discrete metric problems, such as firstpassage percolation (FPP) [62], the actual distance between sites $i$ and $j$ is found by obtaining the discrete geodesic, which we proceed to define. Let $\Gamma$ be any path along the system, $\Gamma=\left\{i_{1}, \ldots, i_{M}\right\}$. Then

$$
D_{i j}=\min _{\Gamma} \sum_{k=1}^{M(\Gamma)-1} d\left(i_{k}, i_{k+1}\right),
$$

where $M(\Gamma)$ is the number of steps of the path $\Gamma$. It is easy to prove that $D_{i j}$ fulfills the distance axioms, including the triangle inequality. Notice that the geodesic can take as many steps as required.

Let us consider a translationally invariant 1D system following the area-law: entanglement across any cut is bounded by a constant, with $J_{i j}=J_{0} \exp (-|i-j| / \xi)$, such as the AKLT state, see Eq. (15). Then, for sites $i<j$ such that $|i-j| \gg \xi$, we see that the geodesic is the straight path $\Gamma=\{i, i+1, \ldots, j\}$, with a distance $D_{i j} \propto|i-j|$. On the other hand, for conformal systems, the straight path competes with the single-step path, which is also of order $D_{i j}=J_{i j}^{-1 / 2} \sim$ $|i-j|$, see Eq. (28).

\section{CONCLUSION AND FURTHER WORK}

To summarize, in this work, we introduced a framework to unveil the geometry suggested by the entanglement structure of any quantum many-body state. The optimal geometry is characterized by the elements of a generalized adjacency matrix, which is obtained by exploring the entanglement entropies computed for all possible bipartitions of the manybody state. We noted that in some cases, the optimal geometry turns out to be completely different from that suggested by the parent Hamiltonian of the model. We later showed how the optimized geometries can provide a natural route to compute the entanglement contour, introduced for noninteracting models. Finally, we showed that for a conformal invariant system, the elements of the generalized adjacency matrices can be related to the two-point correlator of an entanglement current operator. This field theory realization leads to think of entanglement as a flow among the parts of the system, in analogy to the flow of energy that is characterized by the stress tensor. Both entanglement and energy are, after all, fundamental resources of a physical theory [63]. It will also be interesting to analyze the relation of our approach to the geometry proposed for tensor networks [12], to holography in static and dynamic scenarios $[49,64,65]$, and also to a higher-dimension physical system. As a possible application of the formalism to other physical models, we plan to explore quantum disordered and quantum quenched systems in our future works.

\section{ACKNOWLEDGMENTS}

We would like to thank P. Calabrese, J. I. Cirac, J. I. Latorre, E. López, L. Tagliacozzo, E. Tonni, G. Vidal, H. Q. Zhou, Q. Q. Shi, and S.Y. Cho for conversa-

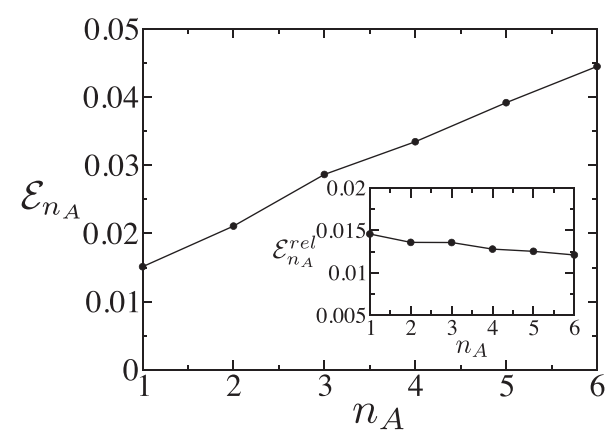

FIG. 9. Plot of distribution of average error $\left(\mathcal{E}_{n_{A}}\right)$ with the number of boundaries between the blocks $\left(n_{A}\right)$, obtained by exploring all possible bipartition of the ground state of the free-fermionic Hamiltonian, defined in Eq. (11) of the main text. In the inset, we also plot the relative error of the entropy $\left(\mathcal{E}_{n_{A}}^{\text {rel }}\right)$, computed using Eq. (A2) with the number of boundaries $\left(n_{A}\right)$. Here $t_{i, i+1}=1$ and $N=14$.

tions. We acknowledge financial support from the Grants No. FIS2015-69167-C2-1-P, No. FIS2015-66020-C2-1-P, No. PGC2018-095862-B-C21, No. PGC2018-094763-B-I00, No. QUITEMAD+ S2013/ICE-2801, No. SEV-2016-0597 of the "Centro de Excelencia Severo Ochoa" Programme and the CSIC Research Platform on Quantum Technologies PTI-001.

\section{APPENDIX A: ESTIMATION OF ERROR AND RANDOM SAMPLING}

In the first part of this section, we provide a more finegrained analysis of the error introduced in the computation of the entropies of each bipartition. In particular, we study how the average error is distributed over different geometries of the bipartitions. Towards this aim, we first group the bipartitions according to the number of boundaries $\left(n_{A}\right)$ they share with the rest of the system. The average error $\left(\mathcal{E}_{n_{A}}\right)$ is then computed using the following relation:

$$
\varepsilon_{n_{A}}=\frac{1}{Z_{A}} \sum_{X \in 0,2^{N-1}}\left|S_{X}-\hat{S}_{X}\right|
$$

where the summation has been taken on the set of bipartitions $X$, which has the same number of boundaries $n_{A}$ and $Z_{A}$ is the cardinality of that set. Figure 9 shows the scaling of the error $\left(\varepsilon_{n_{A}}\right)$ with the number of boundaries $\left(n_{A}\right)$ of the different bipartitions for a free-fermionic model described in Eq. (11) of the main text. From the plot, we can see that the estimated average error is low for bipartitions with a lesser number of boundaries. Now as the bipartitions with more boundaries yield higher entropy values, in the inset, we also provide the relative error introduced in the computation of the entropies. It is defined as

$$
\mathcal{E}_{n_{A}}^{\text {rel }}=\frac{1}{Z_{A}} \sum_{X \in 0,2^{N-1}} \frac{\left|S_{X}-\hat{S}_{X}\right|}{S_{X}} .
$$

We note that the relative error is distributed almost uniformly over bipartitions with all possible geometries.

In the second part of this section, we discuss the efficiency of the random sampling method that has been introduced in Sec. V of the main text. In particular, we compare the behavior 


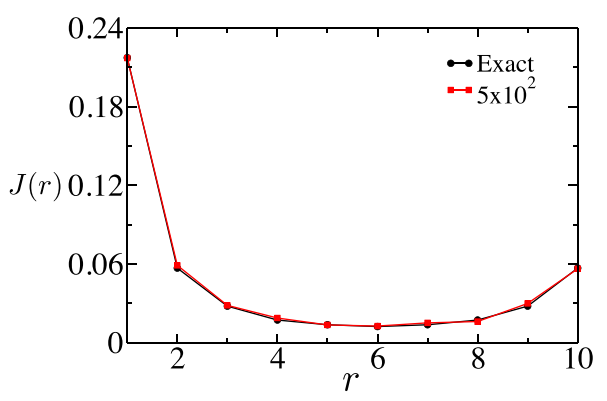

FIG. 10. Comparison of decay of entanglement adjacency matrix $J(r)$ to the site distance $r=|i-j|$ for an $X X Z$ model as given in Eq. (22) of the main text, with $\Delta=0.50$, considering all possible bipartitions (black curve), and for sampling of $5 \times 10^{2}$ number of entropies (red curve). Here $N=12$.

of $J(r)$ to the site distance $r=|i-j|$ that was obtained in the main text by considering all possible bipartitions of the state, to that obtained using a random sampling of less number of entropies. Figure 10 shows one such example where a comparison of $J(r)$ versus the $r$ profile, obtained using the random sampling method, has been made to that of the exact method, for the $X X Z$ model. From the figure, we note that in these cases, a random sampling of a lesser number of entropies can capture all the features of the entanglement adjacency matrix efficiently.

\section{APPENDIX B: LIST OF CONSTRAINTS TO BE FULFILLED BY AN ENTANGLEMENT CONTOUR}

In this section, we discuss two other important properties to be satisfied by the contour function [43] obtained from the entanglement adjacency matrix, as expressed in Eq. (19) in the main text.

(1) Invariance under local unitary transformations: If the unitary transformation $U_{B}$ concerns a region $B$, and the pure state $|\psi\rangle_{A B}$ is related as $\left|\psi^{\prime}\right\rangle_{A B}=U_{B}|\psi\rangle_{A B}$, the entanglement contour functions $s_{A}(i)$ derived for both the states $|\psi\rangle_{A B}$ and $\left|\psi^{\prime}\right\rangle_{A B}$ must be the same.

Since the $J_{i j}$ matrix is obtained from the entanglement entropies of all blocks, this statement is only true for unitary operators $U_{B}$ which act on single sites. In that case, all entropies are invariant under the action of any local unitary operator and, therefore, the $J_{i j}$ matrix inherits that invariance.

(2) (a) Upper bound: If a subregion $A_{1} \subseteq A$ is contained in a factor space $V_{A}=V_{A_{1}} \otimes V_{A_{2}}$ then the entanglement contour of subregion $A_{1}$ cannot be larger than the entanglement entropy $S_{A}$, i.e., $s_{A}\left(A_{1}\right) \leqslant S_{A}$.

For a factorized space such as given above, $V_{A}=V_{A_{1}} \otimes$ $V_{A_{2}}$, the entropy function satisfies the subadditivity condition $S_{A} \leqslant S_{A_{1}}+S_{A_{2}}$. This implies $S_{A_{1}} \leqslant S_{A}$. Again, the entanglement contour of subregion $A_{1}, s_{A}\left(A_{1}\right)=\sum_{i \in A_{1}, j \notin A_{1}} J_{i j}=S_{A_{1}}$. Hence the proof.

Now if we decompose $A_{1}$ further as $V_{A_{1}}=V_{A_{1}^{1}} \otimes V_{A_{1}^{2}}$, the lower bound can be derived as follows.

(b) Lower bound: The entanglement contour of subregion $A_{1}$ is at least equal to the entanglement entropy of $S_{A_{1}^{1}}$.

This we can show again using the subadditivity condition for the above factored space, $S_{A_{1}} \leqslant S_{A_{1}^{1}}+S_{A_{1}^{2}}$. Hence, $S_{A_{1}} \geqslant$
$S_{A_{1}^{1}}$. Now alternatively, we can write $S_{A_{1}}=\sum_{i \in A_{1} j \notin A_{1}} J_{i j}=$ $s_{A}\left(A_{1}\right)$. This implies $s_{A}\left(A_{1}\right) \geqslant S_{A_{1}}$.

\section{APPENDIX C: ENTANGLEMENT CURRENT IN CFT}

Let us consider an interval $A$ of finite length in a larger system. The entanglement entropy $S_{A}$ of the ground state, or the thermal state, is computed using a path integral in a euclidean space-time $\Sigma$. To regularize the path integral one removes infinitesimal discs $D_{\varepsilon}$ (parameterized by a complex coordinate $z$ ) of radius $\varepsilon$, centered around the entangling points of $A$. The resulting space-time $\Sigma \backslash D_{\varepsilon}$, can be mapped, via a conformal transformation $w=f(z)$, into an annulus $\mathbb{A}$ of heigh $2 \pi$ (i.e., $\operatorname{Im} w=\operatorname{Im} w+2 \pi$ ) and width $W_{A}$ given by [51]

$$
W_{A}=\int_{A_{\varepsilon}} d x f^{\prime}(x)
$$

where $A_{\varepsilon}$ denotes the interval left after the removal of the discs $D_{\varepsilon}$. The $n$th Rényi entropy is given by

$$
S_{A}^{(n)}=\frac{c}{12}\left(1+\frac{1}{n}\right) W_{A}+C_{n}+o(1),
$$

where $c$ is the central charge of the CFT and $C_{n}$ is a constant that depends on the boundary entropies and nonuniversal data of the model. A contour is a nonnegative function $s_{A}^{(n)}(x)$ that describes the contribution of the points of the interval $A$ to the $n$th Rényi entropy [43]

$$
S_{A}^{(n)}=\int_{A_{\varepsilon}} d x s_{A}^{(n)}(x), \quad s_{A}^{(n)}(x) \geqslant 0 .
$$

This function is nonunique but Eqs. (C1) and (C2) suggest the ansatz $[24,46]$

$$
s_{A}^{(n)}(x)=\frac{c}{12}\left(1+\frac{1}{n}\right) f^{\prime}(x)+\frac{C_{n}}{\ell},
$$

where $\ell$ is the length of the interval $A$. Our aim is to represent the nonconstant term of the contour function $(\mathrm{C} 4)$ as

$$
s_{A}^{(n)}(x)-\frac{C_{n}}{\ell}=\frac{c}{12}\left(1+\frac{1}{n}\right) f^{\prime}(x)=\int_{\bar{A}} d y J^{(n)}(x, y),
$$

where $\bar{A}$ is the complement of $A$.

The dependence of $J^{(n)}(x, y)$ with respect to $n$ can be factor out defining the function $g(x, y)$,

$$
J^{(n)}(x, y)=\frac{c}{12}\left(1+\frac{1}{n}\right) g(x, y),
$$

that from Eq. (C5) satisfies

$$
f^{\prime}(x)=\int_{\bar{A}} d y g(x, y) .
$$

As an example, let us consider the euclidean space-time with complex coordinate $z=x+i t \in \mathrm{C}$ and the finite interval, at $t=0, A=(u, v) \subset \mathbb{R}$ with $v>u$. Removing two discs of radius $\varepsilon$ around the entangling points $z=u, v$ one obtains the 
I

\section{0}

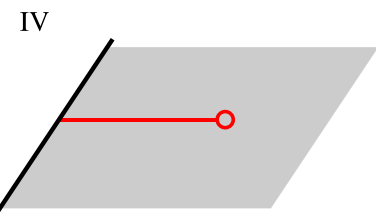

II

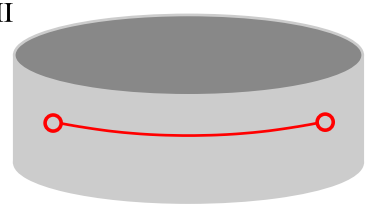

V

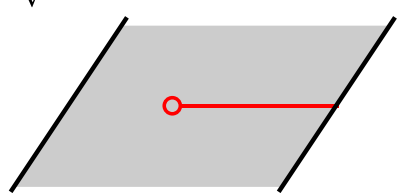

III

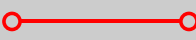

VI

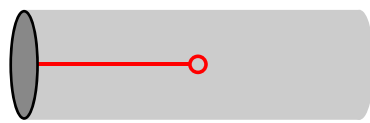

FIG. 11. Euclidean space-time $\Sigma$ domains of the models. The space direction $x$ runs horizontally and the time direction $t$ runs vertically. A red segment denotes the interval $A$ and a red circle the boundary of a disk of infinitesimal radius $\varepsilon$. The boundaries of $\Sigma$ are colored in black. In all these cases, the space-time left after the removal of the disk, or discs, can be mapped to an annulus $\mathbb{A}$, of height $2 \pi$ and width $W_{A}$, by the conformal transformations given in Table I.

annulus $\mathbb{A}$ by the conformal transformation [51]

$$
w=f(z)=\log \frac{z-u}{v-z} .
$$

Choosing the regularized interval as $A_{\varepsilon}=(u+\varepsilon, v-\varepsilon)$ (with $0<\varepsilon \ll 1$ ), one finds from Eqs. (C1) and (C8)

$$
W_{A}=f(v-\varepsilon)-f(u+\varepsilon)=2 \log \frac{v-u}{\varepsilon} .
$$

and from Eq. (C2) the well-known result

$$
S_{A}^{(n)}=\frac{c}{6}\left(1+\frac{1}{n}\right) \log \frac{v-u}{\varepsilon}+C_{n}+o(1) .
$$

To find the function $g(x, y)$, satisfying Eq. (C7), we choose the interval $\bar{A}=(-\infty, u) \cup(v, \infty)$, which does not depend on $\varepsilon$ since the interval $A_{\varepsilon}$ already provides a regularization. Equation (C7) becomes

$$
f^{\prime}(x)=\frac{1}{x-u}+\frac{1}{v-x}=\left(\int_{-\infty}^{u}+\int_{v}^{\infty}\right) d y g(x, y),
$$

and taking a derivative respect to $u$, or $v$, gives

$$
g(x, y)=\frac{1}{(x-y)^{2}}
$$

that together with Eq. (C6) yields Eq. (28). Let us observe that $J(x, y)$ is the two-point correlator of a current $\mathbf{J}(z)$ on the complex plane

$$
J(x, y)=\langle\mathbf{J}(x) \mathbf{J}(y)\rangle_{\text {plane }} .
$$

This method to compute $g(x, y)$ can be applied to the geometries depicted in Fig. 11 where the regularized space-time $\Sigma \backslash D_{\varepsilon}$ is conformally equivalent to an annulus [51]. The results are presented in Table I and using them one can verify Eq. (28) in the main text that generalizes Eq. (C12).

\section{Two disjointed intervals in the infinite line}

Let us apply Eqs. (27) and (28) in the main text to compute the entanglement entropy of the ground state for two disjointed intervals $A=\left(u_{1}, v_{1}\right) \cup\left(u_{2}, v_{2}\right)$ (with $u_{1}<v_{1}<$ $\left.u_{2}<v_{2}\right)$. Choosing the regularization

$$
\begin{aligned}
A_{\varepsilon} & =\left(u_{1}+\varepsilon, v_{1}-\varepsilon\right) \cup\left(u_{2}+\varepsilon, v_{2}-\varepsilon\right), \\
\bar{A} & =\left(-\infty, u_{1}\right) \cup\left(v_{1}, u_{2}\right) \cup\left(v_{2}, \infty\right),
\end{aligned}
$$

one finds (discarding the cutoff $\varepsilon$ )

$$
S_{A}=\frac{c}{3} \ln \left(\frac{\left|u_{1}-v_{1}\right|\left|u_{2}-v_{2}\right|\left|u_{1}-v_{2}\right|\left|u_{2}-v_{1}\right|}{\left|u_{1}-u_{2}\right|\left|v_{1}-v_{2}\right|}\right) .
$$

This results agrees with the one first obtained in Ref. [19]. There is, however, a missing additive term in this expression of $S_{A}$ that depends on the harmonic ratio $x=\left(u_{1}-v_{1}\right)\left(u_{2}-\right.$ $\left.v_{2}\right) /\left[\left(u_{1}-u_{2}\right)\left(v_{1}-v_{2}\right)\right]$, and the operator content of the CFT,

TABLE I. Conformal maps $f(z)$ from the geometries of Fig. 11 into the annulus of widths $W_{A}$ and the corresponding functions $g(x, y)$. In the cases II, III, and V, the length of $A$ is denoted by $\ell$.

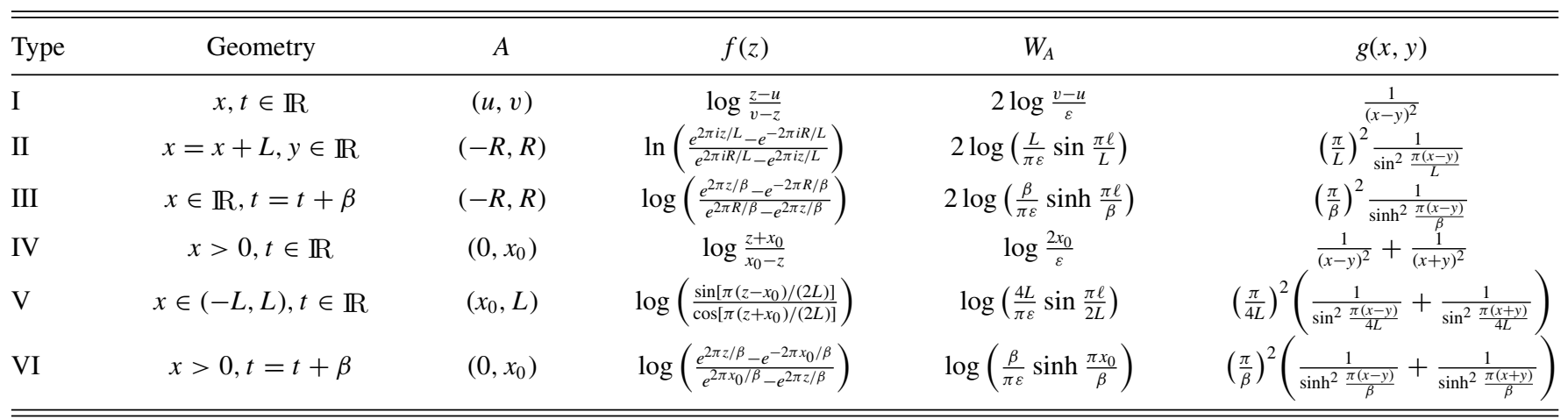


and not only on the central charge [54-57,59,60]. Extensive analytic and numerical work has been devoted to this problem but, to our knowledge, there is not a general analytic formula for $S_{A}$ (see Ref. [60] for a summary and an extended list of references). The generalization of Eq. (C15) to more than two intervals is straightforward.
[1] C. H. Bennett and S. J. Wiesner, Communication Via Oneand Two-Particle Operators on Einstein-Podolsky-Rosen States, Phys. Rev. Lett. 69, 2881 (1992).

[2] C. Bennett, G. Brassard, C. Crepeau, R. Jozsa, A. Peres, and W. Wootters, Teleporting an Unknown Quantum State Via Dual Classical and Einstein-Podolsky-Rosen Channels, Phys. Rev. Lett. 70, 1895 (1993).

[3] R. Horodecki, P. Horodecki, M. Horodecki, and K. Horodecki, Quantum entanglement, Rev. Mod. Phys. 81, 865 (2009).

[4] A. Osterloh, L. Amico, G. Falci, and R. Fazio, Scaling of entanglement close to a quantum phase transition, Nature 416, 608 (2002).

[5] T. J. Osborne and M. A. Nielsen, Entanglement in a simple quantum phase transition, Phys. Rev. A 66, 032110 (2002).

[6] J. M. Maldacena, The Large N limit of superconformal field theories and supergravity, Int. J. Theor. Phys. 38, 1113 (1999).

[7] S. Ryu and T. Takayanagi, Holographic Derivation of Entanglement Entropy from AdS/CFT, Phys. Rev. Lett. 96, 181602 (2006).

[8] G. Vidal, Entanglement Renormalization, Phys. Rev. Lett. 99, 220405 (2007).

[9] M. van Raamsdonk, Building up spacetime with quantum entanglement, Gen. Relativ. Gravit. 42, 2323 (2010).

[10] B. Swingle, Entanglement renormalization and holography, Phys. Rev. D 86, 065007 (2012).

[11] C. Cao, S. M. Carroll, and S. Michalakis, Space from Hilbert space: Recovering geometry from bulk entanglement, Phys. Rev. D 95, 024031 (2017).

[12] K. Hyatt, J. R. Garrison, and B. Bauer, Extracting Entanglement Geometry from Quantum States, Phys. Rev. Lett. 119, 140502 (2017).

[13] L. Amico, R. Fazio, A. Osterloh, and V. Vedral, Entanglement in many-body systems, Rev. Mod. Phys. 80, 517 (2008).

[14] M. Srenidcki, Entropy and Area, Phys. Rev. Lett. 71, 666 (1993).

[15] J. Eisert, M. Cramer, and M. B. Plenio, Colloquium: Area laws for the entanglement entropy, Rev. Mod. Phys. 82, 277 (2010).

[16] M. M. Wolf, F. Verstraete, M. B. Hastings, and J. I. Cirac, Area Laws in Quantum Systems: Mutual Information and Correlations, Phys. Rev. Lett. 100, 070502 (2008).

[17] C. Holzhey, F. Larsen, and F. Wilczek, Geometric and renormalized entropy in conformal field theory, Nucl. Phys. B 424, 443 (1994).

[18] G. Vidal, J. I. Latorre, E. Rico, and A. Kitaev, Entanglement in Quantum Critical Phenomena, Phys. Rev. Lett. 90, 227902 (2003).

[19] P. Calabrese and J. Cardy, Entanglement entropy and quantum field theory, J. Stat. Mech. (2004) P06002.

[20] G. Vitagliano, A. Riera, and J. I. Latorre, Volume-law scaling for the entanglement entropy in spin $1 / 2$ chains, New J. Phys. 12, 113049 (2010).

[21] G. Ramírez, J. Rodríguez-Laguna, and G. Sierra, From conformal to volume-law for the entanglement entropy in exponen- tially deformed critical spin 1/2 chains, J. Stat. Mech. (2014) P10004.

[22] G. Ramírez, J. Rodríguez-Laguna, and G. Sierra, Entanglement over the rainbow, J. Stat. Mech. (2015) P06002.

[23] J. Rodríguez-Laguna, J. Dubail, G. Ramírez, P. Calabrese, and G. Sierra, More on the rainbow chain: Entanglement, spacetime geometry and thermal states, J. Phys. A: Math. Theor. 50, 164001 (2017).

[24] E. Tonni, J. Rodríguez-Laguna, and G. Sierra, Entanglement hamiltonian and entanglement contour in inhomogeneous 1D critical systems, J. Stat. Mech. (2018) 043105.

[25] V. Alba, S. N. Santalla, P. Ruggiero, J. Rodríguez-Laguna, P. Calabrese, and G. Sierra, Usual area-law violation in random inhomogeneous systems, J. Stat. Mech. (2019) 023105.

[26] N. Samos Sáenz de Buruaga, S. N. Santalla, J. RodríguezLaguna, and G. Sierra, Symmetry protected phases in inhomogeneous spin chains, J. Stat. Mech. (2019) 093102.

[27] I. MacCormack, A. Liu, M. Nozaki, and S. Ryu, Holographic duals of inhomogeneous systems: The rainbow chain and the sine-square deformation model, J. Phys. A: Math. Theor. 52, 505401 (2019).

[28] R. Movassagh and P. W. Shor, Power law violation of the area law in quantum spin chains, Proc. Natl. Acad. Sci. USA 113, 13278 (2016).

[29] O. Salberger and V. Korepin, Fredkin spin chains, arXiv:1605.03842.

[30] Z. Zhang, A. Ahmadain, and I. Klich, Novel quantum phase transition from bounded to extensive entanglement, Proc. Natl. Acad. Sci. USA 114, 5142 (2017).

[31] O. Salberger, T. Udagawa, Z. Zhang, H. Katsura, I. Klich, and V. E. Korepin, Deformed fredkin spin chain with extensive entanglement, J. Stat. Mech. (2017) 063103.

[32] F. Sugino and P. Padmanabhan, Area law violations and quantum phase transitions in modified motzkin walk spin chains, J. Stat. Mech. (2018) 013101.

[33] L. Caha and D. Nagaj, The pair-flip model: A very entangled translationally invariant spin chain, arXiv:1805.07168.

[34] F. Sugino and V. Korepin, Renyi entropy of highly entangled spin chains, Int. J. Mod. Phys. B 32, 1850306 (2018).

[35] Since both the empty and the complete block should present zero entropy, thus they will be left out whenever $s_{0} \neq 0$.

[36] M. A. Nielsen and I. L. Chuang, Quantum Computation and Quantum Information, 10th Anniversary Ed. (Cambridge University Press, Cambridge, England, 2010).

[37] I. Affleck, T. Kennedy, E. H. Lieb, and H. Tasaki, Rigorous Results on Valence-Bond Ground States in Antiferromagnets, Phys. Rev. Lett. 59, 799 (1987).

[38] T. Hirano and Y. HatsugaiEntanglement entropy of onedimensional gapped spin chains, J. Phys. Soc. Jpn. 76, 074603 (2007).

[39] H. Katsura, T. Hirano, and V. E. Korepin, Entanglement in an SU(n) valence-bond-solid state, J. Phys. A: Math. Theor. 41, 135304 (2008). 
[40] V. E. Korepin and Y. Xu, Entanglement in valence-bond-solid states, Int. J. Mod. Phys. B 24, 1361 (2010).

[41] I. Peschel, Calculation of reduced density matrices from correlation functions, J. Phys. A: Math. Gen. 36, L205 (2003).

[42] F. Iglói and I. Peschel, On reduced density matrices for disjoint subsystems, Europhys. Lett. 89, 40001 (2010).

[43] Y. Chen and G. Vidal, Entanglement contour, J. Stat. Mech. (2014) P10011.

[44] A. Botero and B. Reznik, Spatial structures and localization of vacuum entanglement in the linear harmonic chain, Phys. Rev. A 70, 052329 (2004).

[45] I. Frérot and T. Roscilde, Area law and its violation: A microscopic inspection into the structure of entanglement and fluctuations, Phys. Rev. B 92, 115129 (2015).

[46] A. Coser, C. D. Nobili, and E. Tonni, A contour for the entanglement entropies in harmonic lattices, J. Phys. A: Math. Theor. 50, 314001 (2017).

[47] Q. Wen, Fine structure in holographic entanglement and entanglement contour, Phys. Rev. D 98, 106004 (2018).

[48] E. Tonni, Entanglement Hamiltonians and Contours on a Segment, Talk at the workshop It from Qubit, Centro Atómico Bariloche, 2018.

[49] J. Kudler-Flam, I. MacCormack, and S. Ryu, Holographic entanglement contour, bit threads, and the entanglement tsunami, J. Phys. A: Math. Theor. 52, 325401 (2019).

[50] In the similar vein as that of the entanglement contour, we propose an ansatz for the elements of the $J$ matrix as follows:

$$
J_{i j}=\left\langle\sum_{p=1}^{\min (|A|,|\bar{A}|)}\left|\Phi_{p, i}^{(A)} \Phi_{p, j}^{(\bar{A})}\right|^{2} H\left(v_{p}\right)\right\rangle,
$$

where $\Phi_{p, j}^{(\bar{A})}$ is the eigenfunction, with eigenvalue $v_{p}$, of the correlation matrix (20) restricted to the block $\bar{A}$, and the average is taken over all bipartitions. Employing (C16) to find $J_{i j}$ presents some numerical instabilities which do not arise when using (17). This equation, however, provides a physical interpretation of $J_{i j}$ for free-fermion models.

[51] J. Cardy and E. Tonni, Entanglement Hamiltonians in twodimensional conformal field theory, J. Stat. Mech. (2016) 123103.
[52] P. Calabrese and J. Cardy, Entanglement entropy and conformal field theory, J. Phys. A 42, 504005 (2009).

[53] J. L. Cardy, O. A. Castro-Alvaredo, and B. Doyon, Form factors of branch-point twist fields in quantum integrable models and entanglement entropy, J. Stat. Phys. 130, 129 (2008).

[54] M. Caraglio and F. Gliozzi, Entanglement entropy and twist fields, J. High Energy Phys. 11 (2008) 076.

[55] S. Furukawa, V. Pasquier, and J. Shiraishi, Mutual Information and Compactification Radius in a $c=1$ Critical Phase in One Dimension, Phys. Rev. Lett. 102, 170602 (2009).

[56] P. Calabrese, J. Cardy, and E. Tonni, Entanglement entropy of two disjoint intervals in conformal field theory, J. Stat. Mech. (2009) P11001.

[57] P. Calabrese, J. Cardy, and E. Tonni, Entanglement entropy of two disjoint intervals in conformal field theory: II, J. Stat. Mech. (2011) P01021.

[58] V. Alba, L. Tagliacozzo, and P. Calabrese, Entanglement entropy of two disjoint blocks in critical Ising models, Phys. Rev. B 81, 060411(R) (2010).

[59] A. Coser, L. Tagliacozzo, and E. Tonni, On Rényi entropies of disjoint intervals in conformal field theory, J. Stat. Mech. (2014) P01008.

[60] P. Ruggiero, E. Tonni, and P. Calabrese, Entanglement entropy of two disjoint intervals and the recursion formula for conformal blocks, J. Stat. Mech. (2018) 113101.

[61] Y. W. Dai, X. H. Chen, S. Y. Cho, H. Q. Zhou, and D. X. Yao, Universal scaling relationship between classical and quantum correlations in critical quantum spin chains, arXiv:1805.03464.

[62] H. Kesten, Percolation theory and first-passage percolation, Ann. Probab. 15, 1231 (1987); P. Córdoba-Torres, S. N. Santalla, R. Cuerno, J. Rodríguez-Laguna, and Kardar-ParisiZhang universality in first passage percolation: The role of geodesic degeneracy, J. Stat. Mech. (2018) 063212.

[63] T. S. Cubitt, F. Verstraete, and J. I. Cirac, Entanglement flow in multipartite systems, Phys. Rev. A 71, 052308 (2005).

[64] J. Abajo-Arrastia, J. Aparicio, and E. Lopez, Holographic Evolution of Entanglement Entropy, J. High Energy Phys. 11 (2010) 149.

[65] M. Freedman and M. Headrick, Bit Threads and Holographic Entanglement, Commun. Math. Phys. 352, 407 (2017). 